\title{
Evolución de las conexiones entre producción y con- sumo y sus impactos en la dinámica de un territorio: el caso de Mocajuba en la Amazonía oriental - PA
}

\author{
Evolution of the connections between production and consumption and \\ their impacts on the dynamics of a territory: the case of Mocajuba in the \\ eastern Amazon - PA
}

Marc Piraux $^{1}$ iD ; Pauline Hélène Cécile Marie Cuenin² iD

\section{RESUMEN}

Debido al alargamiento de las cadenas alimentarias, el sistema alimentario global genera una distancia entre los productores agrícolas y los consumidores. Frente a esta coyuntura global, se destacan los movimientos de resistencia local, que proponen iniciativas de acercamiento entre los agricultores y la sociedad. Así, el objetivo de este trabajo es analizar la evolución de estos procesos de desconexión y reconexión entre producción y consumo, así como las reconfiguraciones territoriales de estos movimientos en las últimas décadas en el municipio de Mocajuba (PA) en la Amazonía Oriental. Para cumplir con el objetivo, se realizó un análisis de los flujos de productos agrícolas y alimenticios entre el municipio y el exterior y dentro del municipio, complementado con un análisis de la trayectoria del territorio en las últimas décadas y proyectos de desarrollo local en curso. Hubo un aumento significativo en el flujo de productos agroindustriales desde fuera del municipio desde los años 2000-2005, específicamente hacia el área rural, posibilitado por programas sociales que elevaron los ingresos de la población. Esto se traduce en una creciente dependencia de estas zonas rurales de los productos agroalimentarios "globalizados". Sin embargo, ha habido movimientos de valorización de productos tradicionales de origen alimentario amazónico como la harina de mandioca y el açaí y, más recientemente, iniciativas de acercamiento entre productores y consumidores de Mocajubes, tendientes a dinamizar las zonas rurales.

Palabras clave: Globalización; Sistemas agroalimentarios; Reconfiguraciones territoriales. Amazonas.

\footnotetext{
Este articulo fue publicado originalmente en REDES (Santa Cruz do Sul. Online), v. 24, n. 3, p. 101-117, setiembre-diciembre, 2019. Fue autorizada por el editor de REDES su publicación en español en la Revista de Investigación Científica y Tecnológica.

${ }^{1}$ Universidad Federal de Pará - Belém - Pará - Brasil

${ }^{2}$ Universidad Federal de Viçosa - Viçosa - Minas Gerais - Brasil

Autor de correspondencia: Marc Piraux. Email: marc.piraux@cirad.fr

Traducción al español realizada por: Dionicio Acuña. Email: dionicio7acuna@hotmail.com
} 


\section{ABSTRACT}

Due to the lengthening of food chains, the global food system creates a distance between agricultural producers and consumers. Faced with this global situation, the local resistance movements stand out, proposing initiatives to bring farmers and society closer together. Thus, the objective of this work is to analyze the evolution of these processes of disconnection and reconnection between production and consumption, as well as the territorial reconfigurations of these movements in recent decades in the municipality of Mocajuba (PA) in the Eastern Amazon. To meet the objective, an analysis of the flows of agricultural and food products between the municipality and abroad and within the municipality was carried out, complemented by an analysis of the trajectory of the territory in recent decades and ongoing local development projects. There was a significant increase in the flow of agroindustrial products from outside the municipality since 20002005 , specifically towards the rural area, made possible by social programs that raised the income of the population. This translates into a growing dependence of these rural areas on "globalized" agri-food products. However, there have been movements of valorization of traditional products of Amazonian food origin such as cassava flour and açaí and, more recently, initiatives of rapprochement between producers and consumers of Mocajubes, aimed at energizing rural areas.

Keywords: Globalization; Agri-food systems; Territorial reconfigurations; Amazon. 


\section{INTRODUCCIÓN}

Desde el inicio de la globalización del sistema agroalimentario, a mediados de los años ochenta, la alimentación ha pasado por un proceso de desarraigo con su base ecológica. Entonces, en un mundo cada vez más globalizado, la sociedad se está desconectando tanto en términos de producción de productos agrícolas como de consumo de alimentos (SCHNEIDER y GAZOLLA, 2017). De hecho, los "imperios alimentarios", que se estructuran como una nueva forma de ordenar el sistema alimentario global, se basan en un modelo de producción que explora la naturaleza y concentra la mayor parte de los mercados agrícolas y alimentarios globales, provocando múltiples desconexiones (VAN DER PLOEG, 2008). En particular, este nuevo sistema alimentario global crea una distancia entre productores y consumidores debido al alargamiento de las cadenas alimentarias. Las cadenas alimentarias largas invisibilizan los alimentos, su identidad, su origen y quién está detrás de cada eslabón de la cadena alimentaria, en particular, el proceso de producción agrícola, siendo una característica del modo industrial de producción de alimentos $(\mathrm{SCH}-$ NEIDER y GAZOLLA, 2017).

Frente a esta coyuntura global, destacan los movimientos de resistencia local, como los procesos de mantenimiento y puesta en valor de los productos locales y las iniciativas de reconexión de los productores de alimentos con los consumidores. Renting, Marsden y Banks (2017, p. 34) enfatizan el papel de las cadenas cortas como "canales para crear nuevos vínculos entre los agricultores y la sociedad, productores y consumidores" en el contexto del desarrollo rural. La agricultura familiar está en el centro de este proceso (VAN DER PLOEG, 2008). La distancia y el acercamiento entre quienes producen y quienes consumen repercuten en los territorios y su dinámica, considerándose un territorio como un espacio apropiado y organizado (MOINE, 2006). La apreciación de los productos alimenticios locales se arraiga en un espacio específico. El territorio presenta así una forma material y simbólica (vivida y representada por los actores del territorio) (RAFFESTIN, 1986).

Sin embargo, el territorio también es un espacio de poder entre actores con intereses diferentes, que tienen la capacidad o no de regular y organizar, para utilizar recursos naturales limitados (DI MEO, 2014). Idealmente, un sistema de gobernanza, basado en una alianza entre la sociedad civil y las autoridades públicas, debe implementar las bases de la acción pública favorable a estas dinámicas de acercamiento entre producción y consumo en un enfoque de desarrollo territorial (PECQUEUR, 2005). Esta se constituye como un proceso de cambio estructural emprendido por una sociedad territorialmente organizada, sustentada en la puesta en valor del capital y los recursos (materiales e inmateriales). Tiene como objetivo impulsar la economía y mejorar la calidad de vida de su población (DalLabrida, 2010).

Estos movimientos de acercamiento entre la producción y el consumo de alimentos tienen una dinámica especial en la Amazonía brasileña. De hecho, está marcado por un gran patrimonio sociocultural que incluye productos alimenticios y culturas específicas, pero también, por ser una zona de disputa de intereses económicos y por dinámicas globalizadas muy intensas que tienen impactos socioespaciales (CASTRO, 2005). Estas dinámicas están particularmente vinculadas a las infraestructuras (puertos, por ejemplo), el desarrollo de los mercados internacionales y la dinámica empresarial, así como a los marcos regulatorios establecidos por las autoridades públicas basados en un marco in- 
ternacional y una agenda política (POLGE et al., 2015). Tan peculiares características hacen de la Amazonía una zona interesante para estudiar la evolución de las configuraciones de vínculos entre agricultores familiares y consumidores y sus impactos en el territorio, directamente influenciados por la globalización.

Queriendo suscribir esta temática, el objetivo de este artículo es analizar la evolución de los procesos de desconexión y reconexión entre la producción y el consumo de alimentos, así como sus reconfiguraciones territoriales en las últimas décadas en el municipio de Mocajuba (PA) en la Amazonas oriental. Este municipio fue elegido por varios criterios: su pequeño tamaño (área), facilitando un análisis de los flujos de recursos y materiales; su gran diversidad socio ecológica; sus varias características tradicionales, en particular, la marcada presencia de la agricultura familiar. Pero, como en el caso de muchos municipios amazónicos (PIRAUX et al., 2015), Mocajuba adolece de una dificultad en la acción colectiva a nivel de agricultura familiar. Queremos mostrar que, en este municipio, ante un proceso de globalización homogeneizadora de los patrones de consumo, existe un movimiento de reconexión entre la producción y el consumo de alimentos, que aún es muy frágil, por la debilidad de la acción colectiva y la dificultad de construir una acción pública de necesaria para neutralizar las acciones de las grandes empresas agroalimentarias.

\section{2 Área de estudio y procedimientos metodológicos}

El municipio de Mocajuba se ubica a $200 \mathrm{~km}$ al sur de Belém, capital del estado de Pará, a orillas del río Tocantins. La población del municipio es de 26.731 habitantes, de los cuales 18.279 en el área urbana y 8.452 en el área rural (IBGE, 2010). $\mathrm{Su}$ superficie es de $870,8 \mathrm{~km}^{2}$. La mayoría de los productos agrícolas que se producen en el municipio provienen de la agricultura familiar muy activa en el territorio (95\% de los agricultores).

Para el estudio, el municipio de Mocajuba fue dividido en cuatro áreas según la zonificación realizada por GEDAF, el Grupo de Estudio sobre la Diversidad de la Agricultura Familiar de la Universidad Federal de Pará - UFPA (PIRAUX et al., 2017) (Figura 1):

- La zona “Quilombola” en la margen derecha del río, en la que la principal producción es la harina de mandioca, siendo la zona más aislada de Mocajuba (Zona 1);

- Las "Islas" del río donde viven los habitantes de la ribera, en las que las principales actividades son la pesca y la extracción (açaí, caucho, cacao, murumuru1, etc.) (Zona 2);

- $\quad$ El área "Tierra Firme", que incluye: un área de cuenca para la producción de harina de yuca (Zona 4) y pimienta negra (cultivo de ingresos) (Zona 5 excluyendo el centro urbanizado) y una pequeña área quilombola con producción diversificada (Zona 3), pero también se centró en la harina de mandioca. Estas zonas se agruparon por proximidad geográfica y estructural;

- El "Centro Urbano" de Mocajuba, esta área fue excluida del área "Tierra Firme" por estar asociada a una dinámica urbana muy diferente a la rural. El principal sector de actividad es el comercio seguido de la industria, la educación y la administración pública (IBGE, 2010).

El mercado municipal y los otros mercados y supermercados de la ciudad son los principales espacios para la compra de alimentos.

1 Palmera nativa del Amazonas cuyo fruto se utiliza generalmente para hacer aceite / mantequilla para uso en cosmética. 
Figura 1: Zonificación en el municipio de Mocajuba

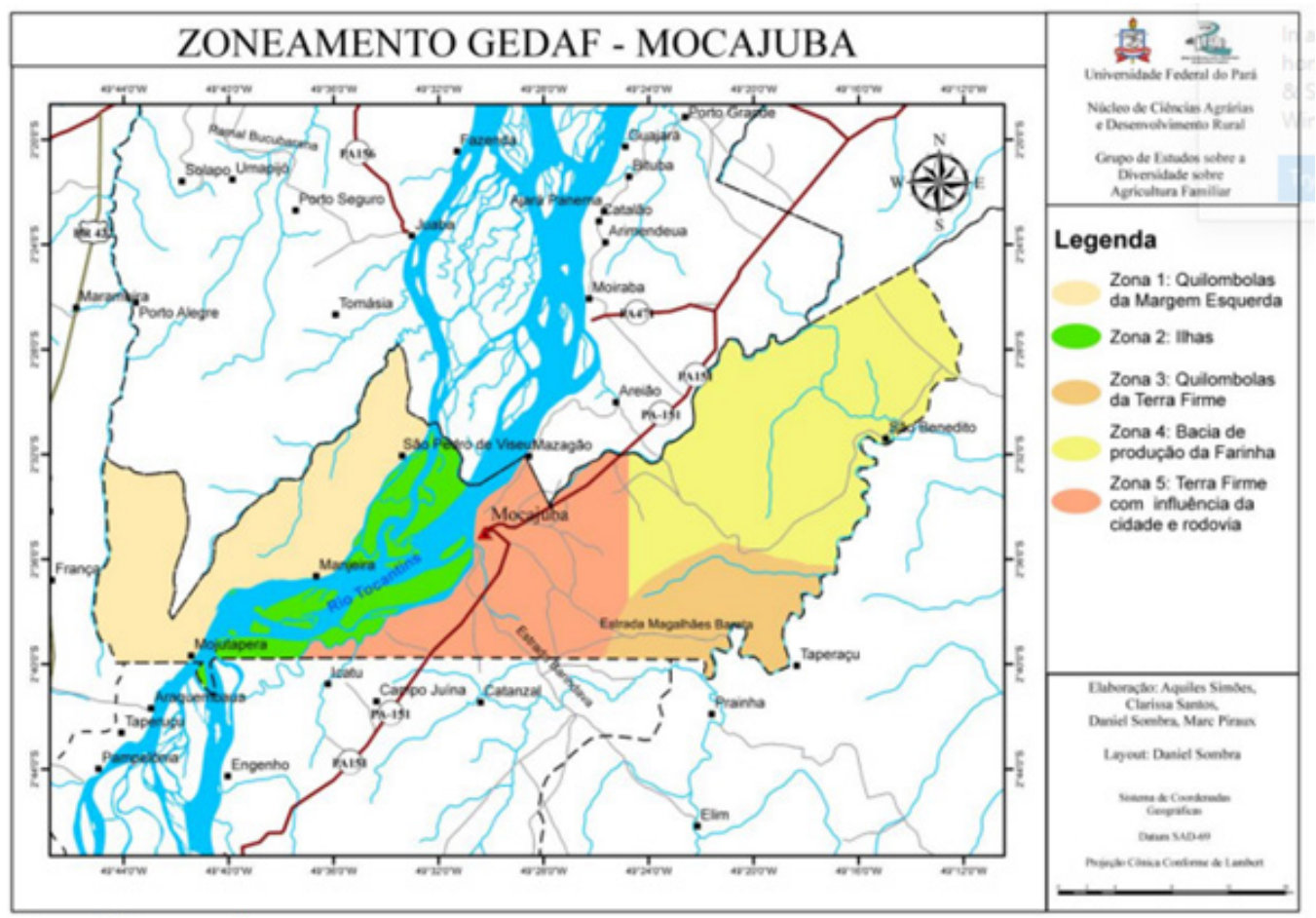

Fuente: Piraux et al., 2017

Para lograr el objetivo de este trabajo se realizó un análisis de los flujos (REYNAUD, 1980) de los principales productos agrícolas y alimenticios entre las distintas áreas del municipio y entre el municipio y el exterior, es decir, los flujos que ingresan y salen del municipio. De hecho, los flujos no ocurren por casualidad, traducen los juegos de los actores. Raffestin (1980), partiendo de una lectura relacional de la organización del mundo, sitúa los flujos en el centro de la producción del espacio humano, es decir, la transformación del espacio en territorio donde "todas las relaciones están marcadas por el poder" (RAFFESTIN, 1980, página 129). La mayoría de estos flujos son imposibles de describir a partir de la información existente, cuyos objetos son otros: uso del suelo, vegetación, etc.

Para ello, se recogieron datos secundarios de diversos documentos disponibles (trabajos de investigación, información de empresas, administraciones públicas, etc.) y datos primarios recogidos a través de entrevistas semiestructuradas y estructuradas con diversos actores del

territorio (agricultores familiares, miembros de la Unión de Trabajadores Rurales - STTR,

Secretario de Agricultura, vendedores responsables y del mercado municipal, gerentes de supermercados, agentes de salud, miembros de diversas asociaciones del municipio, como la Asociación de Asentamiento Ilha da Conceição o la Asociación de Descendientes Quilombolas, etc.). Se evaluaron los grandes tipos de flujos agrícolas y alimentarios y sus lugares de salida y llegada. Valorando el conocimiento local, se realizó un análisis de la trayectoria del territorio en las últimas décadas y de los proyectos de desarrollo local vigentes. Además, se vivió la vida cotidiana de al- 
gunas familias de diferentes áreas rurales y urbanas (una familia por área) con el fin de comprender mejor las relaciones de las personas con la alimentación y las dinámicas presentes en estas áreas. La investigación de campo se realizó en 2014 y se complementó en 2016. Finalmente, se verificó la validez de la información mediante el método de triangulación de las fuentes de información. Se realizó un reembolso a los actores para validar los resultados y complementarlos.

3 De las bases alimentarias tradicionales al incremento de productos agroindustriales y sus consecuencias

Como cultura alimentaria de la población del estado de Pará, el açaí siempre ha sido la base alimentaria de las comunidades ribereñas de Mocajuba, tradicionalmente acompañado de harina de mandioca, pescado y camarones.

El açaí, pescado y camarón, productos que se encuentran espontáneamente en el ambiente de los ríos amazónicos, fueron tradicionalmente extraídos y auto consumidos por estas familias de las "Islas" 2 y una pequeña parte se destinó a la comercialización en el "Centro Urbano" del municipio. La harina de yuca también era un producto alimenticio tradicional en Mocajuba, producido en "Terra Firme" y en la zona de "Quilombola", autoconsumo de las familias de estas zonas rurales.

\subsection{Década de los 80-90: cambios en el} patrón de consumo de alimentos

Sin embargo, esta dinámica tradicional del territorio Mocajuban y el sistema alimentario local asociado se transformó por dos causas principales en la década de 1980. La primera fue la activación de una represa hidroeléctrica en 1985 construida en el río Tocantins aguas arriba de Mocajuba. Esto tuvo un fuerte impacto en los recursos pesqueros del río $\mathrm{y}$, en consecuencia, en la base alimentaria y la vida de las poblaciones ribereñas en las "Islas".
El segundo está asociado con el auge del cultivo de la pimienta negra en la "Tierra Firme" en las décadas de 1970 y 1980, cultura que llegó al municipio en la década de 1960. Se involucra en el negocio (almacenamiento y comercio) de esta cultura, la mayoría de ellos. residiendo en el "Centro Urbano" del municipio. Esto provocó cambios en las necesidades y patrones de consumo de la población.

A principios de la década de los noventa arribaron al mercado de Mocajuba productos agroindustriales de fuera del municipio, como arroz, frijoles, carne de res y pollo.

En la década de 1995, la crisis de la pimienta negra (caída del precio en el mercado internacional y enfermedades fúngicas como la fusariosis del pimiento) provocó el éxodo rural de parte de los agricultores de "Tierra Firme" fuera del municipio o hacia el municipio sede, donde adoptaron los patrones de consumo de alimentos del centro. En el mismo período (1998), la llegada de la energía eléctrica también provocó cambios en los sistemas de consumo, con la compra de productos manufacturados (televisión, frigorífico, etc.), asociados al aumento del consumo de productos agroindustriales.

2 Citamos los nombres de las zonas de zonificación presentadas en el área de estudio entre comillas.

\subsection{Años 2000: programas sociales $y$} homogeneización de patrones culturales y alimentarios

Sin embargo, fue durante la década del 2000 cuando se produjo un crecimiento significativo de los flujos agroindustriales provenientes principalmente de la región de Belém y Castanhal y del ámbito nacional. De hecho, el gobierno federal desarrolló una serie de programas sociales, con énfasis en el programa Bolsa Familia iniciado en 2003, que permitió incrementar los ingresos de las poblaciones principalmente rurales y su acceso a nuevos pro- 
ductos de consumo.

En este proceso, es importante destacar el Decreto No. 6.040 / 2007, que trata del reconocimiento y desarrollo de las poblaciones tradicionales. Este decreto permitió reconocer a las comunidades quilombolas (en el proceso "Tierra Firme" iniciado en 2008 con la recepción del título de territorio quilombola en 2013) y hacer que estas poblaciones y sus territorios sean prioritarios para la aplicación de programas nacionales como el acceso a electricidad, Bolsa Familia etc. La política de reforma agraria del gobierno federal aplicada en el estado de Pará también permitió la creación de un asentamiento agro-extractivo en las "Islas" del municipio, en 2008, del cual cada familia recibió una cantidad de dinero para adquirir las necesidades básicas.

Estos programas nacionales asociados, entre otros, al Seguro Cerrado3 de pesca y retiro rural, permitieron mejorar las condiciones de vida de los habitantes, principalmente en áreas rurales y comunidades tradicionales, y facilitar sus condiciones de acceso a bienes de consumo, sean productos agro-productos industriales o manufacturados. Este acceso también se vio fortalecido por mejoras en las condiciones de infraestructura y transporte (lancha motora, motocicleta) entre las áreas rurales y el centro urbano, y entre el municipio y el exterior.

El fácil acceso y la abundancia de estos productos agroindustriales en el mercado de Mocajuban, dado el desarrollo de los supermercados, provocó cambios profundos en los modos de consumo de alimentos. Pasando cada vez más al consumo de productos agroindustriales, las poblaciones de Mocajuba han reducido considerablemente el consumo de productos de su entorno inmediato, devaluando cada vez más los productos locales. Un residente de las "Islas" de 76 años confirma que hace 30 años: "comíamos pescado, açaí y harina todos los días, frijoles y arroz una vez al año; hoy comemos todos los días". También se informó que en este contexto algunos dejaron de producir harina de yuca en algunas zonas de la "Tierra Firme" y la zona de "Quilombola".

Otro factor relevante en los cambios en los patrones alimentarios está vinculado a la importancia de la información proveniente del nivel nacional e internacional, característica de la globalización hegemónica. Hubo una presencia omnipresente de la televisión en Mocajuba en todas las áreas del territorio y constantes anuncios de coches sonoros en el "Centro Urbano" del municipio. Esta información difunde diferentes imágenes y patrones culturales y publicitarios que promueven el consumo de productos agroindustriales, que obviamente no van acompañados de información sobre sus características, su uso y posibles riesgos para la salud.

Progresivamente, hubo una homogeneización de patrones dietéticos entre el "Centro Urbano" del municipio y las diferentes áreas rurales del territorio Mocajuban. Pero no llegó a todas las áreas del territorio mocajubense. Así, y de manera un tanto paradójica, la marginación de la zona "Quilombola", como consecuencia de su gran distancia de la sede del municipio y el acceso restringido a sus servicios, incluida la televisión, implicó una mejor autonomía alimentaria.

Esta estandarización de la base alimentaria es característica del sistema alimentario global, que genera la reducción de las diferencias culturales y regionales en la alimentación y la dieta (GLIESSMAN, 2015). En general, el aumento en la cantidad de estos productos agroindustriales crea una dependencia creciente de las zonas rurales y sus poblaciones de estos productos alimenticios. Provoca el desarraigo de las poblaciones rurales con su base natural, su cultura alimentaria y su entorno 
local, insertándolas en la lógica del sistema agroalimentario global dominante (SCHNEIDER y GAZOLLA, 2017), fuente de un proceso de desterritorialización (THERY, 2008). Pero también tuvo otros impactos en la salud y el medio ambiente.

$3 \mathrm{El}$ seguro cerrado es un beneficio que otorga el Gobierno Federal a los pescadores artesanales que obtienen ingresos exclusivamente de la pesca. Por tanto, se trata de una especie de "seguro de desempleo" para el pescador, al que se le impide pescar en determinadas épocas del año, para garantizar la conservación del pescado.

De hecho, en las zonas rurales de Mocajuba se aprecia cada vez más productos alimenticios como refrescos o galletas industriales, que son bajos en nutrientes y con altos riesgos para la salud (SCHNEIDER y GAZOLLA, 2017). El alto consumo de estos productos por parte de los niños genera muchos problemas dentales y de obesidad, que se ven agravados por las dificultades de acceso a los centros de salud. Estos problemas de salud fueron confirmados por los servicios de salud del municipio.

Otra consecuencia negativa de este cambio en el patrón de consumo se refiere a la alta cantidad de residuos en el medio ambiente, producto del volumen de envases generalmente plásticos para productos agroindustriales y la mala gestión de la recogida de residuos en las zonas rurales. Las entrevistas y la experiencia en las familias confirmaron que la población sigue arrojando estos bultos al medio ambiente como si se tratara de cualquier residuo orgánico sin ser consciente del daño que ocasionan al medio ambiente.

4 La valorización de los productos alimenticios amazónicos: entre estrategias de resistencia, amenazas y riesgos

En los últimos años (años 2010-2015) se pudo observar que, a pesar de la integración de la mayoría de las áreas rurales del municipio al sistema alimentario global, algunos productos alimenticios tradicionales amazónicos se destacaron por ser valorados por varios actores locales. Estas nuevas dinámicas corresponden a estrategias para reactivar recursos locales específicos (PECQUEUR, 2005).

\subsection{La producción de harina de man- dioca de calidad: una estrategia de re- sistencia}

El cultivo de mandioca para su transformación en harina sigue siendo una de las principales actividades agrícolas en una parte del área "Tierra Firme" y el área "Quilombola", siendo producida para autoconsumo y fuente de ingresos. Sousa y Piraux (2015) enfatizaron que la venta de harina de yuca de las comunidades "quilombolas", que se encuentran marginadas, ha ganado protagonismo en los últimos años. Los consumidores del "Centro Urbano" incluyen a los municipios, reconociendo la alta calidad del producto y la compra. Otras comunidades, como Baratinha, ubicada en "Tierra Firme", también buscaron mejorar la calidad del proceso de transformación de la yuca. Las adaptaciones y cambios en las prácticas asociadas a este proceso fueron el resultado de una serie de intercambio de información y experimentación por parte de los propios agricultores (SOUSA y PIRAUX, 2015). El objetivo también era desarrollar un nicho de mercado en el "Centro Urbano" de Mocajuba y así resolver las dificultades en la comercialización de la harina, que efectivamente es el caso. El control de calidad se ha convertido en una nueva norma para las comunidades rurales.

Estos son ejemplos de estrategias de resistencia campesina contra las fuerzas del sistema alimentario global. De hecho, según Van Der Ploeg (2008, 2009), esta resistencia se da en varios niveles y en diversas formas, construyendo una multiplicidad de respuestas que reconectan personas, actividades y proyectos. 
Permiten establecer o mantener nuevas conexiones con el consumidor, dando visibilidad al producto, aportando su propia identidad y aumentando la autonomía de los productores, contribuyendo así a un proceso de reterritorialización (VAN DER PLOEG, 2008). Estas estrategias de resistencia y acercamiento con los consumidores locales son importantes para generar un nuevo dinamismo en las zonas rurales. Sin embargo, también es importante destacar las amenazas externas a estos procesos.

\subsection{La amenaza de la agricultura in- dustrial}

En primer lugar, se observaron amenazas por la llegada a la "Tierra Firme" de grandes terratenientes que invierten y se apoderan de grandes extensiones, para desarrollar el cultivo de pimienta negra, açaí de regadío o ganadería, generando, entre otros, competencia en el uso del agua con los agricultores familiares, fuente de algunos conflictos. Esta apropiación y degradación de los recursos naturales es, en general, un tipo de amenaza externa a la agricultura familiar que destaca Van Der Ploeg (2014). Por ejemplo, en la "Tierra Firme" de Mocajuba, se informó de un caso conflictivo entre un gran terrateniente y una comunidad quilombola vecina. Los habitantes de esta comunidad observaron una disminución en la cantidad de agua en el río debido a las prácticas de riego en el cultivo de açaí. Estos conflictos sociales influyen directamente en la producción de los agricultores familiares. También hubo dificultades de acceso físico a la tierra para algunos agricultores familiares debido a la apropiación de una gran cantidad de tierra por parte de grandes productores rurales.

Además, la implementación de cultivos básicos comerciales de destino nacional e internacional puede competir con cultivos tradicionales basados en alimentos y / o devaluar los conocimientos tradicionales de los agricultores familiares. De hecho, los sistemas de producción de estos cultivos se basan en las prácticas y padrones estandarizados del régimen socio-técnico de la agricultura industrial guiados por los valores de mercado y los principios de la ciencia moderna: agricultura intensiva, aplicación de fertilizantes sintéticos, riego, químicos. control de plagas mediante el uso de plaguicidas y manipulación del genoma vegetal (GLIESSMAN, 2015). Son sistemas de producción cada vez más especializados y artificializados, que provocan varias desconexiones (VAN DER PLOEG, 1992; VAN DER PLOEG et al., 2004). En particular, hay un alejamiento de la naturaleza y una ruptura con la dinámica del mantenimiento del equilibrio ecológico y con las prácticas y conocimientos locales (CAPORAL y COSTABEBER, 2007).

En la "Tierra Firme" de Mocajuba, el cultivo de la pimienta negra y, sobre todo, la palma aceitera, son los dos principales cultivos basados en estos principios. El proyecto BBB (Belém Bioenergia Brasil, formado por Petrobras y la empresa portuguesa Galp) inició en 2013 el cultivo de palma aceitera en propiedades de agricultores familiares del municipio.

El objetivo inicial fue la producción de biodiesel a partir de un estímulo de políticas públicas (Pronaf Eco-dendê). La implementación de este proyecto estuvo acompañada de la implementación de reglas de gestión y el uso de paquetes tecnológicos impuestos por las empresas. La salida completa de la BBB en 2016, debido a la falta de rentabilidad y el difícil acceso al municipio (presencia de un ferry en un río en la carretera principal a Belém), dejó a los agricultores familiares comprometidos con la empresa, sin apoyo y con un futuro incierto. Algunos agricultores también han reducido la producción de yuca.

Gliessman (2015) ya llamó la atención sobre el hecho de que, dada la presión del sistema alimentario global para plan- 
tar cada vez más cultivos comerciales, se reduce el consumo de productos locales y las ventas en los mercados locales. Los sistemas agrícolas asociados a estos cultivos generan la devaluación de los conocimientos tradicionales de los agricultores familiares, que sin embargo son importantes para la autonomía de las familias agrícolas y para la construcción de sistemas alimentarios alternativos.

Otra amenaza externa sobre los sistemas productivos de la agricultura familiar y sobre el potencial de iniciativas de valoración de productos tradicionales está relacionada con la volatilidad de los mercados agrícolas y la fluctuación en los precios de venta de los productos (VAN DER PLOEG, 2014). Cuando aumentan los precios de los cultivos comerciales, se anima a los agricultores a dejar de cultivar productos alimenticios básicos. Por ejemplo, en Mocajuba, ha habido una reanudación del cultivo de la pimienta negra en los últimos años debido al aumento de su precio de venta, lo que repercute en las zonas de producción de yuca, cuyo cultivo se considera más doloroso.

\subsection{El desarrollo del açaí en las "Islas":} entre resistencia y estrategia de riesgo

En el proceso de valoración de los productos alimenticios amazónicos, además de la harina de yuca, el desarrollo del cultivo del açaí es otro fenómeno interesante a mencionar en el territorio Mocajuban. El cultivo de açaí de várzea es tradicional en las "Islas" y es representativo del agroextractivismo y la cultura ribereña de esta zona. El cultivo de açaí ha aumentado significativamente en los últimos años en respuesta al crecimiento de la demanda regional, nacional e internacional. En los últimos diez años, ha habido un flujo de ventas creciente desde las "Islas" hacia el "Centro Urbano" de Mocajuba. Allí, se consume directamente o se vende a través de intermediarios al mercado regional. En Mocajuba, la producción está en el dominio de los ribereños y no es impuesta por agentes externos. Esta valorización de los recursos locales permite dinamizar la zona de las "Islas" del municipio, y esta actividad es una fuente de ingresos muy importante.

Sin embargo, varios riesgos están asociados con este proceso. Primero, para responder a una demanda creciente del mercado nacional o internacional, el açaí tiende a ingresar a largas cadenas alimentarias, en las que el patrón de producción evoluciona como una "mercancía": estandarización de prácticas (monocultivo y riego), búsqueda de rentabilidad financiera, productivismo y, al final, pérdida de identidad del producto a lo largo de la cadena. Además, Homma et al. (2006) demostraron que la expansión del consumo de pulpa de açaí promueve la exclusión social de los consumidores con menor poder adquisitivo que tradicionalmente eran los principales consumidores. Este es el caso de algunos agricultores ribereños de Mocajubense que venden su producción de açaí para comprar productos agroindustriales.

En la "Tierra Firme" de Mocajuba, ya se puede ver el florecimiento de las áreas de cultivo de açaí de regadío, desconectando esta planta de su entorno y manejo tradicional. Con la creciente demanda y los programas del gobierno del estado de Pará para incrementar la producción de açaí, debe incrementarse la implementación de nuevas áreas de açaí de regadío en la "Tierra Firme", pudiendo competir con el resto de productos alimenticios. de las familias rurales.

En definitiva, la valorización de los productos tradicionales, ya sea açaí o mandioca, son importantes para reconectar al agricultor y al consumidor con su entorno y su cultura local, impulsando así las zonas rurales productoras. Sin embargo, son 
necesarias muchas precauciones para no caer en la lógica de los "imperios alimentarios", que tienden a alargar las cadenas de suministro y eliminar el conocimiento, la memoria y la identidad de los productos alimenticios.

En vista de la riqueza de los recursos locales de las "Islas", otros productos del agroextractivismo podrían ser valorados por la gente de ribera, a través del reconocimiento del origen y calidad de los productos alimenticios, como es el caso del cacao. Los procesos de valorización del cacao aún enfrentan varias dificultades. En 2013, el comprador de cacao del municipio inició un proceso para valorar este producto a través de la certificación orgánica en conjunto con algunos agricultores, considerando una demanda de una cooperativa Castanhal. El intento fue un fracaso ante las dificultades burocráticas del proceso que enfrentan los agricultores, la dificultad de la acción colectiva y la falta de capacitación de los líderes locales, propia de esta zona (PIRAUX et al., 2015). Porque los líderes locales tienen un papel importante como agentes de cambio (ROEPE y WISKERKE, 2004). Permiten impulsar cambios movilizando e iniciando acciones colectivas y, así, creando estrategias innovadoras a nivel local.

5 iniciativas para reconectar la producción local con los consumidores de Mocajuban

En Mocajuba se observaron otras iniciativas que van más allá de la valorización de los productos alimentarios tradicionales amazónicos y que permiten reconectar a productores y consumidores del municipio. Destaca el flujo de productos alimenticios locales entre las distintas zonas de Mocajuba. Estos flujos de baja intensidad (en volumen y valor monetario) corresponden a iniciativas tomadas con el propósito de valorizar e incrementar la producción local para abastecer también la demanda local. Se pueden destacar varias iniciativas.

A partir de 2016, cabe destacar un dinamismo en la implementación del Programa Nacional de Alimentación Escolar (PNAE), un programa de gran interés para fortalecer la producción familiar local (Resque et al., 2019), lo que conlleva una reducción de la dependencia de los alimentos. A través del PNAE, la Secretaría de Agricultura del municipio intentó iniciar proyectos de cría de peces y distribución de semillas de frijol con los agricultores de "Tierra Firme" para promover la producción local. Otros productos locales como la yuca y sus derivados, la maracuyá, el jerimum, por ejemplo, fueron parte del programa. Además, el PNAE, al apoyar la comercialización de pollos de corral, estimuló la creación de pollos de corral, que se desarrolló en el municipio por iniciativa de algunos ganaderos. Aunque las cantidades vendidas en el mercado son mínimas, en comparación con el volumen de pollos industriales, esta experiencia es un ejemplo concreto de iniciativas locales, con potencial de crecimiento en el municipio. En la misma perspectiva de aglutinar a productores y consumidores agrícolas en Mocajubes, el Sindicato de Trabajadores Rurales (STTR) comenzó a promover productos locales (hortalizas y frutas) con la realización de ferias agroecológicas4 en 2016.

Sin embargo, estas iniciativas de reconexión enfrentan muchas dificultades. En primer lugar, la segunda edición de la feria agroecológica fracasó por un motivo que planteó STTR: tanto para los agricultores como para los consumidores, existe una falta de información y conocimiento sobre la importancia de valorar los productos locales. La movilización de productores y consumidores fue difícil. Según un miembro del STTR, los agricultores no se daban cuenta de que tenían productos que po- 
dían vender. Esta misma dirección sindical observó que los consumidores de la feria fueron aquellos con mayor nivel educativo (médicos, docentes, etc.), quienes consideran que estos productos son de mayor calidad. Los demás consumidores prefirieron comprar frutas al por menor, incluso con precios iguales a los productos de la feria.

Como señala Gliessman (2015), los sistemas alimentarios globales, al desconectar los procesos de producción de la distribución, aíslan a los consumidores de la información necesaria para obtener un mayor conocimiento sobre la importancia de la elección de alimentos.

Por parte de los productores, la concentración de poder de los "Imperios Alimentarios" hace que los productos de la agricultura familiar sean generalmente despreciados, considerados fuera de los estándares del sistema alimentario global (VAN DER PLOEG, 2009). Muchos consumidores y jóvenes entrevistados destacan este hecho.

Con el fin de informar, sensibilizar y empoderar a más agricultores y consumidores sobre el tema, STTR en alianza con UFPA y la Federación de Organismos de Asistencia Social y Educativa (FASE)5, comenzó a organizar talleres y seminarios sobre diversos temas relacionados con la agroecología. Comenzó con talleres con grupos de mujeres. FASE, junto con la red de la Asociación Pará de Apoyo a las Comunidades Necesitadas (APACC), que tiene su sede en el municipio vecino, organizan proyectos y acciones sobre agroecología y economía solidaria en la región, como el Foro de Economía Solidaria y proyectos" Zona quilombola" de Mocajuba. Así, el reforzamiento de estos temas en Mocajuba y el inicio de la estructuración de un movimiento agroecológico dentro de las redes agroecológicas regionales es una señal prometedora para desarrollar iniciativas de reconexión de la producción local con los consumidores de Mocajuba.

Pero, en general, estas iniciativas también enfrentan muchas dificultades. La primera dificultad está ligada a los precios bajísimos de los productos agroindustriales que compiten de manera desigual con los productos de la agricultura familiar. De hecho, en el contexto del monopolio de poder de los "imperios alimentarios", es mucho más difícil, tanto para los agricultores como para los consumidores, salir de los circuitos controlados por ellos (VAN DER PLOEG, 2009). Así, para mantener el monopolio en el mercado, una posible estrategia de las grandes empresas vinculadas a las cadenas de supermercados es bajar los precios de sus productos para ser aún más competitivos en relación a los productos de la agricultura familiar. En Mocajuba, este fenómeno ocurrió luego de la introducción en el mercado local de gallinas de corral criadas por agricultores familiares, un proyecto iniciado por uno de los ex secretarios de agricultura y que fracasó por la caída de los precios de los pollos industriales. Esto muestra la dificultad de crear un sistema de gobernanza territorial que involucre a todos los actores locales, incluidos los privados.

$4 \mathrm{La}$ agroecología se refiere a acciones que promueven cambios en las dimensiones ecológica, económica y social del sistema agroalimentario para lograr un sistema más sustentable y justo (GLIESSMAN, 2018). En la feria agroecológica, hay productos que se originan en sistemas de producción basados en el conocimiento de la población local, sobre la diversidad biológica y cultural del sitio de producción con mínimo impacto en el medio ambiente pero no necesariamente con certificación orgánica que se rige por normas de producción específica.

5 FASE es una organización no gubernamental, fundada en 1961, cuya misión 
es contribuir a la construcción de una sociedad democrática y activa a favor de alternativas al modelo de desarrollo actual, trabajando en el fortalecimiento de los grupos sociales para garantizar los derechos, la democracia y la sostenibilidad (FASE, 2019).

Esta dificultad está directamente asociada con el contexto político local. La existencia de procesos judiciales vinculados a problemas de corrupción que involucran al Ayuntamiento, provocó una fuerte inestabilidad política. En el momento de la investigación, el alcalde se vio obligado a dejar su cargo después de dos años de batalla legal, regresando al cargo en un momento posterior y dejándolo nuevamente poco tiempo después. Esto explica la desorganización de los servicios municipales y el no funcionamiento del Consejo Municipal de Desarrollo Rural Sostenible (CMDRS), principal órgano de gobierno de los municipios para el área rural. Esto también debilitó la implementación del PNAE en los años 2010-2015 hasta las recientes iniciativas destacadas anteriormente.

\section{Consideraciones finales}

En el municipio de Mocajuba, la globalización alimentaria se tradujo, desde los años 2000-2005, en la expansión de la cantidad de productos agroindustriales ofertados en el mercado local. Los programas sociales, cuya población se ha beneficiado, han facilitado el acceso a estos productos.

La intensa llegada de estos productos de cadena larga a las zonas rurales del municipio ha generado procesos de desvalorización de los productos locales y elementos vinculados a ellos, como la identidad cultural y el saber hacer. La globalización también ha reconfigurado otras dinámicas locales, aumentar los flujos de salida del municipio de productos locales como el açaí, alimento básico de las poblaciones ribereñas. Estos procesos provocaron un distanciamiento de las poblaciones rura- les con los productos alimenticios tradicionales locales y un cambio en el patrón de consumo de alimentos, consecuencia de la intensificación de la circulación de productos agroindustriales e información que transmite un nuevo modelo cultural alimentario que parece negar la territorialidad. La homogeneización de este patrón alimentario entre las distintas áreas del municipio se ha convertido en una realidad. Podemos hablar de un proceso de desterritorialización (THERY, 2008).

En contraste con este proceso hegemónico impuesto por el sistema alimentario global, se han desarrollado estrategias de resistencia por parte de los agricultores familiares. Intentan revalorizar los productos locales tradicionales y reconectar a los productores locales con los consumidores de Mocajuba. Aparecen nuevas transmisiones. La calidad de los productos se convierte en una nueva norma, abarcando los procesos de producción y transformación, como es el caso de la harina de yuca y la calidad sanitaria de los alimentos en el contexto de los productos agroecológicos y de corral. Sin embargo, identificamos amenazas y riesgos a este proceso de reterritorialización, impuesto por el régimen dietético hegemónico que tienden a disminuir e incluso bloquear la creación de experiencias innovadoras (VAN DER PLOEG et al., 2004). Estas amenazas, combinadas con un contexto político local desfavorable, limitaron el impacto de estas iniciativas.

Las iniciativas innovadoras identificadas en el campo, aunque aún sean de pequeño alcance, frágiles y sujetas a muchas amenazas, tienden a reconfigurar las cadenas de suministro de alimentos. Crean nuevos vínculos entre los agricultores y la sociedad y acercan a una parte de los consumidores a los alimentos, sus características y su ubicación. Esto fue posible gracias a la capacidad de resiliencia y articulación 
de varios actores locales, quienes impulsaron nuevos tipos de proyectos de desarrollo rural basados en la valorización de los productos locales. Esta afirmación converge con las ideas de Goodman (2017) y Renting, Marsden y Banks (2017) que demuestran que la reconfiguración de las cadenas de suministro subyace en el surgimiento de nuevas prácticas de desarrollo rural. Sin embargo, estos proyectos afectan principalmente a la zona rural de "Tierra Firme", dejando de lado la zona de "Quilombola", que siempre ha estado más aislada de diversos procesos.

Hacer frente a la diversidad de las zonas rurales se convierte en un desafío importante. Por ello, cabe destacar la importancia de considerar las especificidades ecológicas y culturales de cada zona del municipio en la construcción de estrategias de reconexión entre la producción y el consumo de alimentos saludables y sostenibles.

Para impulsar el surgimiento de nuevas estrategias son necesarias políticas públicas específicas, como las relacionadas con la capacitación y seguimiento o los programas institucionales de compra de alimentos. Pero para mejorar la efectividad de estas políticas públicas, deben combinarse con el fortalecimiento de la acción colectiva y la capacidad organizativa, dando lugar a una acción pública de calidad. Vimos en nuestro caso que la gobernanza territo- rial, es decir, la capacidad de una sociedad organizada territorialmente para gestionar y controlar los asuntos públicos era muy débil. Pero sería necesario mejorar la regulación de las acciones de las grandes empresas y el acceso a los recursos (tierra, agua), cuyo uso es cada vez más tenso en el contexto de la globalización. Esta necesaria articulación entre territorios y el funcionamiento de cadenas productivas se convierte en un importante desafío de investigación y desarrollo.

En general, las perspectivas multinivel y multiactor, propias del desarrollo territorial, se vuelven imprescindibles para la construcción de nuevas experiencias locales y sistemas alimentarios alternativos. En este sentido, el refuerzo en la construcción de la red territorial agroecológica en formación, que incluye varios tipos de actores, es relevante para repensar los sistemas agroalimentarios, los modelos clásicos de desarrollo territorial y para estimular múltiples estrategias innovadoras, revalorizando las prácticas locales de los agricultores y pueblos tradicionales, específicamente en lo que se refiere al agroextractivismo.

Estos procesos deben ser impulsados desde los marcos políticos y regulatorios locales, regionales y nacionales, en sentido contrario a la actual deconstrucción de muchas políticas públicas de fortalecimiento de la agricultura familiar.

\section{REFERENCIAS}

1. CAPORAL, F. R.; COSTABEBER, J. A. Agroecologia: Conceitos e Princípios para a Construção de Estilos de Agriculturas Sustentáveis. In: CAPORAL, F. R. (Orgs.). Extensão Rural e Agroecologia: temas sobre um novo desenvolvimento rural, necessário e possível. Brasilia: MDA, 2007, p. 225-240
2. CASTRO, E. Dinâmica socioeconômica e desmatamento na Amazônia, Novos Cadernos NAEA, v. 8, n. 2, p. 5-39, dez. 2005

3. DALlABRIDA, V. R. Desenvolvimento e Governança territorial: um ensaio preliminar sobre a necessidade da 
regulação no processo de gestão do desenvolvimento. REDES, v. 15 , n. 3 , p. $165-$ 186, 2010.

4. DI MEO, G. Introduction à la géographie sociale. Paris: Arnaud Collin, 2014. FASE. Missão e estratégia. 2019. Disponível em: <https://fase.org.br/pt/ quem-somos/missao-e-estrategia/>. Acesso em: 10 de junho de 2019.

5. GLIESSMAN, S. R. Agroecology : The Ecology of Sustainable Food Systems. Taylor \& Francis Group, 2015.

6. GLIESSMAN, S.Agroecology and SustainableFoodSystems.Agroecologyand

Sustainable Food Systems, v. 42, n. 6, p. 599-600, 2018. Disponível em: <https://doi.org/10.1080/21683565.2018.143 2329>. Acesso em: 10 de junho de 2019.

\section{GOODMAN, D. Espaço} e lugar nas redes alimentares alternativas: conectando produção e

consumo. In: SCHNEIDER, S.; GAZOLLA, M. (Orgs.). Cadeias curtas e redes agroalimentares alternativas: negócios e mercados da agricultura familiar. Porto Alegre: Editora da UFRGS, 2017, p. 59-82.

8. HOMMA, A. K. O., NOGUEIRA, O. L., MENEZES, A. J. E. A., CARVALHO, J. E. U., NICOLI, C. M. L., MATOS, G. B. Açaí : novos desafios e tendências. Amazônia: ciência e desenvolviemento, v. 1, n. 2, p. 7-23, 2006

9. IBGE. Mocajuba. 2010. Disponível em: <https://cidades.ibge.gov.br/brasil/pa/mocajuba/panorama $>$. Acesso em: 9 de novembro de 2017

10. MOINE, A. Le territoire comme un système complexe. L'espace géographique, v. 2, n. 35, p. 115-132, 2006.

11. PECQUEUR, B. Desenvolvimento territorial: uma nova abordagem dos processos de desenvolvimento para as eco- nomias do Sul. Raízes, v. 24, n. 1 e 2, p. 10-22, 2005

12. Piraux M., Cialdella N., Poccard-Chapuis R., Assis W. O futuro incerto da agricultura familiar na amazônia brasileira: um desafio para territórios e políticas públicas. In: MIRANDA C., GUIMARÃES I. (Orgs.). Agricultura familiar, ruralidade, território e política pública. (Serie DRS vol 23). Brasília: IICA, 2015, p. 85-92.

13. Piraux M., Sombra Soares D., Simoes A. A diversidade socioespacial do território baixo tocantins e impactos na agricultura familiar. In: SIMÕES A., BENASSULY M. (Orgs.). Na várzea e na terra firme: transformações socioambientais e reinvenções camponesas. Belém: Universidade Federal do Pará, 2017, p. 77114.

14. Polge, E., Poccard-Chapuis, R., Piraux, M., Territoires émergents d'Amazonie: analyse comparée des dynamiques territoriales dans le Baixo Amazonas et le Nordeste Paraense. Confins, v. 24, 2015. Disponível em: < http://confins.revues. org/10290>. Acesso em: 16 de fevereiro de 2019.

15. RAFFESTIN, C. Ecogénèse territoriale et territorialité. In: AURIAC, F.; BRUNET, R. (Orgs.). Espaces, jeux et enjeux. Paris: Fayard, 1986, p. 175-185.

16. RAFFESTIN, C. Pour une géographie du pouvoir. Paris: Libraires techniques, 1980.

17. RENTING, H.; MARSDEN, T.; BANKS, J. Compreendendo as redes alimentares alternativas: o papel de cadeias curtas de abastecimento de alimentos no desenvolvimento rural. In: SCHNEIDER, S.; GAZOLLA, M. (Orgs.). Cadeias curtas e redes agroalimentares alternativas: negócios e mercados da agricultura familiar. 
Porto Alegre: Editora da UFRGS, 2017, p. 27-52.

18. Resque A.G.L., Coudel E., Piketty M.G., Cialdella N., Sá T., Piraux M., Assis W., Le Page C. 2019. Agrobiodiversity and public food procurement programs in Brazil: Influence of local stakeholders in configuring green mediated markets. Sustainability, v. 11, n. 5, 2019. Disponível em: $<$ https://doi.org/10.3390/su11051425>. Acesso em: 26 de junho de 2019.

19. SCHNEIDER, S.; GAZOLLA, M. Cadeias curtas e redes agroalimentares alternativas. In: SCHNEIDER, S.; GAZOLLA, M. (Orgs.). Cadeias curtas e redes agroalimentares alternativas: negócios e mercados da agricultura familiar. Porto Alegre: Editora da UFRGS, 2017, p. 9-24.

20. SMITH, C.; BRAGDON, S. H. Small-scale farmer innovation. Geneva: Quaker United Nations Office, 2015.

21. SOUSA, F. F. De; PIRAUX, M. A construção social da qualidade da farinha de mandioca em comunidades rurais na Amazônia paraense. Novos Cadernos NAEA, v. 18, n. 3, p. 199-222, 2015.

22. THERY H. Mondialisation, déterritorialisation, reterritorialisation. Bulletin de l'Association e Géographes Français, v. 85, n. 3, p. 324-331, 2008.

23. VAN DER PLOEG, J. D. El processo de trabajo agrícola y la mercantilización. In: GUZMAN, E. S. (Orgs.). Ecología, Campesinado e Historia. Madrid: Ediciones, 1992, p. 163-195.

24. VAN DER PLOEG, J. D. BOUMA, J., RIP, A., RIJKENBERG, F. H. J., VENTURA, F., WISKERKE, J. S. C. On regimes, novelties, niches and co-production. In: WISKERKE, J. S. C.; PLOEG, J. (Orgs.). Seeds of Transition. Essays on novelty production, niches and regimes in agriculture, 2004.

25. VAN DER PlOEG, J. D. Camponeses e Impérios Alimentares. Porto Alegre: UFRGS,

2008.

26. VAN DER PLOEG, J. D. Sete teses sobre a agricultura camponesa. Revistas Agriculturas: Experiências em Agroecologia, n. Especial, p. 17-32, 2009.

27. VAN DER PLOEG, J. D. Cadernos de dabate : Dez qualidades da agricultura familiar. Agriculturas, n. 1, p. 1-16, 2014.

\section{BIOGRAFÍA}

\section{Marc Piraux}

Doutor em Agro-economia pela Faculté des sciences agronomiques de Gembloux (FUSAGX), Bélgica. Pesquisador do Centre de coopération International em Recherche Agronomique pour le Développement (CIRAD). Profesor da Universidade Federal do Pará (UFPA). Marc.piraux@cirad.fr

\section{Pauline Hélène Cécile Marie Cuenin}

Mestre em Agronomia e Meio-Ambiente no Institut National Supérieur des Sciences Agromiques. Mestranda em Extensão Rural na Universidade Federal de Viçosa (UFV). pauline.cuenin@ufv.br 